PROCEEDINGS OF THE

AMERICAN MATHEMATICAL SOCIETY

Volume 132, Number 9, Pages 2497-2503

S 0002-9939(04)07544-6

Article electronically published on April 21, 2004

\title{
EIGENVALUE FIELDS OF HYPERBOLIC ORBIFOLDS
}

\author{
EMILY HAMILTON AND ALAN W. REID
}

(Communicated by Linda Keen)

\begin{abstract}
In this paper, we prove that if $\Gamma$ is a non-elementary subgroup of $\mathrm{O}_{\mathrm{o}}(n, 1, \mathbb{R})$, with $n \geq 2$, then the eigenvalue field of $\Gamma$ has infinite degree over $\mathbb{Q}$.
\end{abstract}

\section{INTRODUCTION}

We begin by recalling some basic notions. We shall denote hyperbolic $n$-space by $\mathbb{H}^{n}$. For $n \geq 2$, the group of isometries of $\mathbb{H}^{n}$ can be identified with $\mathrm{O}_{\mathrm{o}}(n, 1, \mathbb{R})$, the subgroup of index two in $\mathrm{O}(n, 1, \mathbb{R})$ that transforms positive time-like vectors into positive time-like vectors. By a non-elementary subgroup of $\mathrm{O}_{\mathrm{o}}(n, 1, \mathbb{R})$ we mean a subgroup that has more than two limit points for its action on the sphere-at-infinity, and by a hyperbolic n-orbifold we mean a quotient $\mathbb{H}^{n} / \Gamma$, where $\Gamma$ is a discrete subgroup of $\mathrm{O}_{\mathrm{o}}(n, 1, \mathbb{R})$. Therefore, given a hyperbolic $n$-orbifold $M=\mathbb{H}^{n} / \Gamma$ with $n \geq 2$, there exists a discrete faithful representation

$$
\rho: \Gamma \rightarrow \mathrm{O}_{\mathrm{o}}(n, 1, \mathbb{R}) .
$$

We make the following definition:

Definition 1.1. If $\Gamma$ is a subgroup of $\operatorname{GL}(n, \mathbb{C})$, let $\mathbb{Q}(e v \Gamma)$ denote the field obtained by adjoining the eigenvalues of the elements of $\Gamma$ to $\mathbb{Q}$, and let $\mathbb{Q}(\operatorname{tr} \Gamma)$ denote the field obtained by adjoining the traces of the elements of $\Gamma$ to $\mathbb{Q}$.

In this paper we consider eigenvalue fields when $\Gamma$ is a subgroup of $\mathrm{O}_{\mathrm{o}}(n, 1, \mathbb{R})$. In the special case when $M=\mathbb{H}^{n} / \Gamma(n \geq 3)$ has finite volume, by Mostow Rigidity, the representation $\rho$ is unique up to conjugation, and we may define the $\mathrm{O}_{\mathrm{o}}(n, 1, \mathbb{R})$ eigenvalue field of $M$ to be $\mathbb{Q}(\operatorname{ev}(\rho(\Gamma))$. One motivation for studying such fields was [9], which showed:

Theorem 1.2. Let $\Gamma$ be a non-elementary subgroup of $\operatorname{PSL}(2, \mathbb{C})$. Then $\mathbb{Q}(\operatorname{ev} \Gamma)$ has infinite degree over $\mathbb{Q}$.

The proof of Theorem 1.2 uses the solution to the Mordell conjecture given by Faltings. We present an elementary proof of Theorem 1.2 in this paper, which allows for considerable generalization.

Received by the editors January 15, 2001.

2000 Mathematics Subject Classification. Primary 20H10; Secondary 20 G30.

The first author was partially supported by NSF Grant DMS 9973317.

The second author was partially supported by the NSF and the Alfred P. Sloan Foundation. 
Specifically, we prove:

Theorem 1.3. Let $\Gamma$ be a non-elementary subgroup of $\mathrm{O}_{o}(n, 1, \mathbb{R})$ with $n \geq 2$. Then $\mathbb{Q}(\mathrm{ev} \Gamma)$ has infinite degree over $\mathbb{Q}$.

As a corollary of this we have the following:

Corollary 1.4. Let $M$ be a finite volume hyperbolic $n$-orbifold with $n \geq 3$. Then the $\mathrm{O}_{\mathrm{o}}(n, 1, \mathbb{R})$ eigenvalue field of $M$ has infinite degree over $\mathbb{Q}$.

Note that in contrast, by Mostow Rigidity, if $n \geq 3$ and $M=\mathbb{H}^{n} / \Gamma$ has finite volume, then $\mathbb{Q}(\operatorname{tr} \rho(\Gamma))$ has finite degree over $\mathbb{Q}$.

\section{Preliminaries}

In this section we collect results that will be needed in the proof of Theorem 1.3

2.1. We begin with the following, which is well known in the case of finite covolume groups (see [12], Proposition 6.6), but in this generality appears not to be in the literature.

Theorem 2.1. Let $\Gamma$ be a finitely generated non-elementary subgroup of $\mathrm{O}_{\mathrm{o}}(n, 1, \mathbb{R})$ whose traces consist of algebraic numbers. Then $\Gamma$ is conjugate in $\mathrm{O}_{\mathrm{o}}(n, 1, \mathbb{R})$ to a group whose elements have entries in a number field.

The proof of Theorem 1.3 does not logically need the full statement of Theorem 2.1, since for the proof it suffices to work with complex representations. For example, given the hypothesis of Theorem [2.1] we can apply [1] to provide a conjugation in $\operatorname{GL}(n+1, \mathbb{C})$ so that the matrices have algebraic entries. However, this conjugation need not be in $\mathrm{O}_{\mathrm{o}}(n, 1, \mathbb{R})$, and it is not clear how to promote this argument to work in the group $\mathrm{O}_{\mathrm{o}}(n, 1, \mathbb{R})$.

Theorem 2.1 seems interesting in its own right and we give a proof below. This will require some notions from representation varieties. For details see [5] and [8].

The group $G=\mathrm{SO}(n, 1, \mathbb{R})$ can be viewed as the $\mathbb{R}$-points of a complex algebraic group $\mathbf{G}=\mathrm{SO}(n, 1, \mathbb{C})$. We will use $G_{\mathrm{o}}$ to denote the identity component of $G$. Let $\Gamma$ be a finitely generated group, and let $\operatorname{Hom}(\Gamma, G)$ denote the set of representations of $\Gamma$ into $G$. Then $\operatorname{Hom}(\Gamma, G)$ is the real points of the complex algebraic variety $\operatorname{Hom}(\Gamma, \mathbf{G})$ (which is defined over $\mathbb{Q}$ ). Now $\mathbf{G}$ acts algebraically on $\operatorname{Hom}(\Gamma, \mathbf{G})$ by conjugation, this action being denoted by $g . \rho$. This action yields a quotient variety, denoted by $X(\Gamma, \mathbf{G})$, which is defined over $\mathbb{Q}$. Let $\pi: \operatorname{Hom}(\Gamma, \mathbf{G}) \rightarrow X(\Gamma, \mathbf{G})$ denote the quotient map, which is also defined over $\mathbb{Q}$. The complex algebraic group $\mathrm{O}(n, 1, \mathbb{C})$ also acts on $\operatorname{Hom}(\Gamma, \mathbf{G})$ by conjugation giving a further quotient variety, denoted by $X^{\prime}(\Gamma, \mathbf{G})$, and a map $\pi^{\prime}$ (both also defined over $\mathbb{Q}$ ). Hence, there is a map

$$
r: X(\Gamma, \mathbf{G}) \rightarrow X^{\prime}(\Gamma, \mathbf{G}),
$$

also defined over $\mathbb{Q}$ with $\pi^{\prime}=r \circ \pi$. Lemma 3.1 of $[8]$ shows that the trace functions generate the coordinate ring of $X^{\prime}(\Gamma, \mathbf{G})$. With this discussion we deduce:

Corollary 2.2. Let $\rho \in \operatorname{Hom}(\Gamma, \mathbf{G})$ be such that $\rho(\Gamma)$ has algebraic traces. Then $\pi(\rho) \in X(\Gamma, \mathbf{G})$ has algebraic coordinates.

Proof. Since traces generate the coordinate ring of $X^{\prime}(\Gamma, \mathbf{G}), \pi^{\prime}(\rho)$ has algebraic coordinates. The map $r$ is defined over $\mathbb{Q}$, and since $\pi(\rho) \in r^{-1}\left(\pi^{\prime}(\rho)\right)$ the result follows. 
We will also make use of the following (see [5], Lemma 3.3 and [8], Corollary $1.2)$.

Lemma 2.3. Let $\rho_{1}, \rho_{2} \in \operatorname{Hom}(\Gamma, G)$, and assume $\rho_{1}(\Gamma)$ is Zariski dense. Then $\pi\left(\rho_{1}\right)=\pi\left(\rho_{2}\right)$ if and only if $\rho_{1}$ and $\rho_{2}$ are conjugate in $G$.

Lemma 2.4. Let $\rho \in \operatorname{Hom}(\Gamma, G)$ with image contained in $G_{\mathrm{O}}$. Then the orbit $\mathbf{G} . \rho$ is closed in $\operatorname{Hom}(\Gamma, \mathbf{G})$, unless $\rho(\Gamma)$ is contained in a parabolic subgroup of $G$.

Proof of Theorem 2.1, We begin with a well-known lemma.

Lemma 2.5. Let $\Gamma<G_{\mathrm{O}}$ be a finitely generated Zariski dense subgroup of $G$ consisting of matrices having algebraic entries. Let $x \in \mathrm{O}_{\mathrm{o}}(n, 1, \mathbb{R})$ normalize $\Gamma$. Then $x$ has algebraic entries.

Proof. Since $\Gamma$ is finitely generated, we can assume that the field generated by the entries of $\Gamma$ is a number field, $k$ say. Thus $\Gamma<M(n+1, k)$, and since $\Gamma$ is Zariski dense in $G, \Gamma$ contains a $k$-basis for $M(n+1, k)$. Since $x$ normalizes $\Gamma$, we can extend the conjugation action of $x$ using the basis above and obtain an automorphism $\phi_{x}: M(n+1, k) \rightarrow M(n+1, k)$, which is given by $\phi_{x}(a)=x a x^{-1}$. Since a matrix algebra is a central simple algebra, any such automorphism is inner by the Skolem-Noether Theorem. Thus there exists $y \in \mathrm{GL}(n+1, k)$ such that

$$
\phi_{x}(a)=y a y^{-1}
$$

for all $a \in M(n+1, k)$. Thus we have

$$
y a y^{-1}=x a x^{-1}
$$

for all $a \in M(n+1, k)$. Thus $x$ differs from $y$ by an element of the center of $M(n+1, k)$, that is, $x=d y$ for some $d \in k$. Hence the entries of $x$ are algebraic numbers.

We now commence with the proof. By Lemma 2.5 it suffices to prove the result for $\Gamma<G_{\mathrm{o}}$.

Thus we assume $\Gamma<G_{\mathrm{o}}$, and let $\rho$ denote the identity representation of $\Gamma$ into $G_{\mathrm{o}}$. By assumption $\Gamma$ is non-elementary and so is not contained in a parabolic subgroup. We can assume that $\Gamma$ is Zariski dense, for if not, then since $\Gamma$ is nonelementary, it follows from the classification of Lie subgroups of $\mathrm{O}(n, 1, \mathbb{R})$ that the group leaves invariant a totally geodesic submanifold of $\mathbf{H}^{n}$ (see [3], Theorems 4.4.1 and 4.4.2). Thus $\Gamma<\mathrm{O}_{\mathrm{o}}(m, 1, \mathbb{R})$ for some $m<n$. Now $\Gamma$ need not lie in the group $\mathrm{SO}_{\mathrm{o}}(m, 1, \mathbb{R})$. However, we can apply the argument below to a subgroup of finite index of $\Gamma$ and then finish off the argument for $\Gamma$ using Lemma [2.5.

By Lemma [2.4, G. $\rho$ is a complex algebraic set. We are assuming that $\Gamma$ has algebraic traces, which are real by assumption on $\Gamma$. Therefore, by Corollary 2.2 $\pi(\rho)$ has real algebraic coordinates. As remarked above, $\pi$ is defined over $\mathbb{Q}$, thus G. $\rho=\pi^{-1}(\pi(\rho))$ is defined over a real number field $k$ and $\mathbf{G} . \rho \cap \operatorname{Hom}(\Gamma, G)$ is a nonempty real algebraic set defined over $k$. To complete the proof we make use of the following consequence of the Tarski-Seidenberg Principle (see 2], Chapters 1 and 4):

Theorem 2.6. Let $U$ be a nonempty real semi-algebraic set defined over a number field $K$. Then $U$ contains a point with algebraic coordinates. 
By Lemma 2.3, any representation in $\operatorname{Hom}(\Gamma, G) \mathbf{G}$-conjugate to $\rho$ is $G$-conjugate to $\rho$. Thus the representation produced by Theorem 2.6 gives the desired conjugate group in $G$. To pass to a $G_{\mathrm{O}}$-conjugate we note the following. Suppose that $A \in G$ is such that $A \Gamma A^{-1}<\mathrm{SO}(n, 1, k)$ for the real number field $k$ produced above. We can assume that $A \notin G_{0}$. Now choose any $B \in \mathrm{SO}(n, 1, k) \backslash \mathrm{SO}_{0}(n, 1, k)$ (such an element exists by standard properties of the spinor norm, see [11]). Then $B A \in \mathrm{SO}_{0}(n, 1, k)$ has the property that $B A \Gamma(B A)^{-1}<\mathrm{SO}_{0}(n, 1, k)$. This yields the final conjugation.

2.2. We will assume standard terminology and results from algebraic number theory. For reference, see [4]. We fix the following notation:

Notation 2.7. (1) By a number field we mean a finite field extension of $\mathbb{Q}$. If $k$ is a number field, let $\mathcal{O}$ denote the ring of integers of $k$. If $\mathfrak{p}$ is a nonzero prime of $\mathcal{O}_{k}$, then we complete $k$ at $\mathfrak{p}$ to obtain the local field $k_{\mathfrak{p}}$, with ring of integers $\mathcal{O}_{k_{\mathfrak{p}}}$. The ring $\mathcal{O}_{k_{\mathfrak{p}}}$ has a unique maximal ideal. The quotient of $\mathcal{O}_{k_{\mathfrak{p}}}$ by this maximal ideal is the residue class field of $\mathcal{O}_{k_{\mathfrak{p}}}$.

(2) A prime $p \in \mathbb{Z}$ splits completely in $k$ if the principal ideal in $\mathcal{O}_{k}$ generated by $p$ factors as a product of $n$ distinct prime ideals of $\mathcal{O}_{k}$, where $n$ is the degree of $k$ over $\mathbb{Q}$. If $p$ splits completely in $k$, then the residue class field of $\mathcal{O}_{k_{\mathfrak{p}}}$ is the finite field consisting of $p$ elements.

2.3. We need the following fact from the theory of finite orthogonal groups. For a discussion, see [6] or [11.

Theorem 2.8. Let $F$ be a finite field of odd characteristic, and let $\Omega(n, 1, F)$ denote the commutator subgroup of $\mathrm{O}(n, 1, F)$. Then $\mathrm{O}(n, 1, F) / \Omega(n, 1, F) \cong \mathbb{Z} / 2 \mathbb{Z} \oplus \mathbb{Z} / 2 \mathbb{Z}$.

2.4. The connection between the preliminaries discussed here is the following. Suppose that $\Gamma$ is a discrete subgroup of $\mathrm{O}_{\mathrm{o}}(n, 1, \mathbb{R})$ of finite co-volume with $n \geq 2$, and that the trace of each element of $\Gamma$ is an algebraic number (this is automatic if $n \geq 3)$. By Theorem 2.1 we can conjugate so that $\Gamma \subset \mathrm{O}_{\mathrm{o}}(n, 1, k)$, where $k$ is a number field. Choose $k$ to be a minimal such field. Let $R$ denote the ring generated by the coefficients of the generators of $\Gamma$ over $\mathbb{Z}$. Since $\Gamma$ has finite co-volume, $R$ is finitely generated. Therefore, $R \subset \mathcal{O}_{k_{\mathfrak{p}}}$ for all but a finite number of primes $\mathfrak{p}$ of $k$. For each of these primes $\mathfrak{p}$, let $F_{\mathfrak{p}}$ denote the residue class field of $\mathcal{O}_{k_{\mathfrak{p}}}$, and let

$$
\pi_{\mathfrak{p}}: \mathcal{O}_{k_{\mathfrak{p}}} \rightarrow F_{p}
$$

denote the residue map. This map induces a group homomorphism

$$
\rho_{\mathfrak{p}}: \mathrm{O}\left(n, 1, \mathcal{O}_{k_{\mathfrak{p}}}\right) \rightarrow \mathrm{O}\left(n, 1, F_{\mathfrak{p}}\right) .
$$

The following result follows from work of Weisfeiler [13] or Nori [10]. For a proof in this setting, see Theorem 2.6 of [6].

Theorem 2.9. In the notation above, for all but a finite number of primes $\mathfrak{p}$ of $k$,

(1) $\Omega\left(n, 1, F_{\mathfrak{p}}\right) \subset \rho_{\mathfrak{p}}(\Gamma) \subset \mathrm{O}\left(n, 1, F_{\mathfrak{p}}\right)$, when $n$ is even, and

(2) $P \Omega\left(n, 1, F_{\mathfrak{p}}\right) \subset \mathrm{P} \rho_{\mathfrak{p}}(\Gamma) \subset \mathrm{PO}\left(n, 1, F_{\mathfrak{p}}\right)$, when $n$ is odd.

\section{Proof of Theorem 1.3}

We begin with the proof of Theorem 1.2, since it illustrates the key ideas involved in the proof of Theorem 1.3 
Theorem 1.2. Let $\Gamma$ be a non-elementary subgroup of $\operatorname{PSL}(2, \mathbb{C})$. Then $\mathbb{Q}(\operatorname{ev} \Gamma)$ has infinite degree over $\mathbb{Q}$.

Proof. Let $\Gamma$ be a non-elementary subgroup of $\operatorname{PSL}(2, \mathbb{C})$. By replacing $\Gamma$ with a finitely generated, non-elementary subgroup, if necessary, we assume that $\Gamma$ is finitely generated. Suppose that $\mathbb{Q}(\mathrm{ev} \Gamma)$ is a number field $F$. Then $\mathbb{Q}(\operatorname{tr} \Gamma) \subset$ $\mathbb{Q}(\mathrm{ev} \Gamma)=F$. By Proposition 2.2(e) of [1], we may conjugate $\Gamma$ in $\operatorname{PSL}(2, \mathbb{C})$ to lie in a finite field extension of $\mathbb{Q}(\operatorname{tr} \Gamma)$. Therefore, we view $\Gamma \subset \operatorname{PSL}(2, k)$, where $k$ is a finite field extension of $F$. Let $R$ be the ring generated by the coefficients of the generators of $\Gamma$ over $\mathbb{Z}$. Since $R$ is finitely generated, $R \subset \mathcal{O}_{k_{\mathfrak{p}}}$ for all but a finite number of primes $\mathfrak{p}$ of $k$. For each of these primes $\mathfrak{p}$, let $F_{\mathfrak{p}}$ denote the residue class field of $\mathcal{O}_{k_{\mathfrak{p}}}$, and let

$$
\pi_{\mathfrak{p}}: \mathcal{O}_{k_{\mathfrak{p}}} \rightarrow F_{p}
$$

denote the residue map. This map induces a group homomorphism

$$
\rho_{\mathfrak{p}}: \operatorname{PSL}\left(2, \mathcal{O}_{k_{\mathfrak{p}}}\right) \rightarrow \operatorname{PSL}\left(2, F_{\mathfrak{p}}\right) .
$$

By the proof of Theorem 1.2 of [7, there exist infinitely many primes $\mathfrak{p}$ of $k$, such that $\rho_{\mathfrak{p}}(\Gamma)=\operatorname{PSL}\left(2, F_{\mathfrak{p}}\right)$. Fix one of these primes such that $F_{\mathfrak{p}}$ has odd characteristic. Choose $b \in F_{\mathfrak{p}}$ such that $b^{2}-4$ is not a square in $F_{\mathfrak{p}}$. This is possible since $F_{\mathfrak{p}}=\left\{4 x \mid x \in F_{\mathfrak{p}}\right\}$, and not every element of $F_{\mathfrak{p}}$ is a square. Then the characteristic polynomial of

$$
A=\left(\begin{array}{cc}
0 & -1 \\
1 & b
\end{array}\right) \in \operatorname{PSL}\left(2, F_{\mathfrak{p}}\right)
$$

is irreducible over $F_{\mathfrak{p}}$. By our choice of $\mathfrak{p}$, there exists an element $\gamma \in \Gamma$ such that $\rho_{\mathfrak{p}}(\gamma)=A$. Let $S$ denote the set of eigenvalues of $\gamma$. Since each eigenvalue of $\gamma$ is a root of a monic polynomial with coefficients in $R, S$ is integral over $R$. It follows that $S \subset \mathcal{O}_{k_{\mathrm{p}}}$. Therefore, the characteristic polynomial of $\gamma$ splits completely over $\mathcal{O}_{k_{\mathfrak{p}}}$. But then the characteristic polynomial of $\rho_{\mathfrak{p}}(\gamma)=A$ splits completely over $F_{\mathfrak{p}}$, a contradiction.

We now proceed with the proof of Theorem 1.3. We prove it in a special case first and then discuss the modifications needed to complete the proof.

Theorem 3.1. Let $\Gamma$ be a discrete subgroup of $\mathrm{O}_{\mathrm{o}}(n, 1, \mathbb{R})$ of finite co-volume with $n \geq 2$. Then $\mathbb{Q}(\mathrm{ev} \Gamma)$ has infinite degree over $\mathbb{Q}$.

Proof. Let $\Gamma$ be a discrete subgroup of $\mathrm{O}_{\mathrm{o}}(n, 1, \mathbb{R})$ of finite co-volume with $n \geq$ 2. Note that if there is a transcendental trace, the eigenvalue field is of infinite degree. Therefore, we can assume that the traces are algebraic - this is automatic in dimensions $\geq 3$ by Mostow Rigidity. By Theorem 2.1. we can conjugate so that $\Gamma \subset \mathrm{O}_{\mathrm{o}}(n, 1, k)$, where $k$ is a number field. Choose $k$ to be a minimal such field.

Suppose that $\mathbb{Q}(\operatorname{ev}(\Gamma))$ is a number field $F$. Let $L$ be the compositum of $F$ and $k$. By adjoining $i \sqrt{2}$ to $L$, if necessary, we assume that $i \sqrt{2} \in L$. Let $R$ be the ring generated by the coefficients of the generators of $\Gamma$ over $\mathbb{Z}$. Since $\Gamma$ has finite co-volume, $R$ is finitely generated. Therefore, $R \subset \mathcal{O}_{k_{\mathfrak{p}}}$ for all but a finite number of primes $\mathfrak{p}$ of $k$. For each of these primes $\mathfrak{p}$, let $F_{\mathfrak{p}}$ denote the residue class field of $\mathcal{O}_{k_{\mathfrak{p}}}$, and let

$$
\pi_{\mathfrak{p}}: \mathcal{O}_{k_{\mathfrak{p}}} \rightarrow F_{\mathfrak{p}}
$$


denote the residue map. This map induces a group homomorphism

$$
\rho_{\mathfrak{p}}: \mathrm{O}\left(n, 1, \mathcal{O}_{k_{\mathfrak{p}}}\right) \rightarrow \mathrm{O}\left(n, 1, F_{\mathfrak{p}}\right) .
$$

By the Tchebotarev Density Theorem [4], there exist infinitely many primes $p$ of $\mathbb{Z}$ that split completely in $L$. By Theorems 2.8 and 2.9, we may fix one of these primes $p$, with extension $\mathfrak{p}$ in $\mathcal{O}_{k}$, such that $\left\{g^{4} \mid g \in \mathrm{O}\left(n, 1, F_{\mathfrak{p}}\right)\right\} \subset \rho_{\mathfrak{p}}(\Gamma)$. Moreover, we may assume that $p \neq 2$.

Let $\mathfrak{P}$ be the extension of $p$ in $\mathcal{O}_{L}$, and let $E_{\mathfrak{P}}$ denote the residue class field of $\mathcal{O}_{L_{\mathfrak{P}}}$. Consider the following diagram:

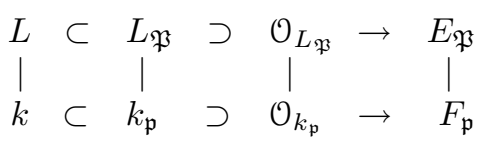

Since $p$ splits completely in $L, E_{\mathfrak{P}}$ is the finite field of $p$ elements. Since $F_{\mathfrak{p}}$ is a subfield of $E_{\mathfrak{P}}$ of characteristic $p$, this implies that $F_{\mathfrak{p}}=E_{\mathfrak{P}}$. As before, choose $b \in F_{\mathfrak{p}}$ such that $b^{2}-4$ is not a square in $F_{\mathfrak{p}}$. Since $i \sqrt{2} \in L,-2$ is a square in $E_{\mathfrak{P}}=F_{\mathfrak{p}}$. As is easily checked,

$$
B=\left(\begin{array}{ccc}
b \sqrt{-2} & b^{2}+1 & b^{2} \\
1 & -b \sqrt{-2} & -b \sqrt{-2} \\
b \sqrt{-2} & b^{2} & b^{2}-1
\end{array}\right) \in \mathrm{O}\left(2,1, F_{\mathfrak{p}}\right) .
$$

Hence

$$
A=\left(\begin{array}{cc}
I_{n-2} & 0 \\
0 & B
\end{array}\right) \in \mathrm{O}\left(n, 1, F_{\mathfrak{p}}\right)
$$

where $I_{n-2}$ is the $(n-2) \times(n-2)$ identity matrix. The characteristic polynomial of $A^{4}$ is

$$
f(t)=(-1)^{n+1}(t-1)^{n-1}\left(t^{2}+\left(-b^{8}+8 b^{6}-20 b^{4}+16 b^{2}-2\right) t+1\right) .
$$

The discriminant of $t^{2}+\left(-b^{8}+8 b^{6}-20 b^{4}+16 b^{2}-2\right) t+1$ is $\left(b^{2}-4\right) b^{2}\left(b^{2}-2\right)^{2}$. $\left(b^{4}-4 b^{2}+2\right)^{2}$. By assumption, $b^{2}-4$ is not a square in $F_{\mathfrak{p}}$. Therefore, $t^{2}+$ $\left(-b^{8}+8 b^{6}-20 b^{4}+16 b^{2}-2\right) t+1$ is irreducible in $F_{\mathfrak{p}}[t]$. We conclude that $f(t)$ does not split completely over $F_{\mathfrak{p}}$. By our choice of $\mathfrak{p}$, there exists an element $\gamma \in \Gamma$ such that $\rho_{\mathfrak{p}}(\gamma)=A^{4}$. Let $S$ denote the set of eigenvalues of $\gamma$. Since each eigenvalue of $\gamma$ is a root of a monic polynomial with coefficients in $R, S$ is integral over $R$. Since $R \subset \mathcal{O}_{k_{\mathfrak{p}}}, S \subset L \subset L_{\mathfrak{P}}$, and $\mathcal{O}_{L_{\mathfrak{P}}}$ is the integral closure of $\mathcal{O}_{k_{\mathfrak{p}}}$ in $L_{\mathfrak{P}}$, this implies that $S \subset \mathcal{O}_{L_{\mathfrak{p}}}$. Therefore, the characteristic polynomial of $\gamma$ splits completely over $\mathcal{O}_{L_{\mathfrak{P}}}$. But then the characteristic polynomial of $\rho_{\mathfrak{P}}(\gamma)=A^{4}$ splits completely over $E_{\mathfrak{P}}=F_{\mathfrak{p}}$, a contradiction.

The proof of the main theorem is completed as follows.

For a general non-elementary subgroup, as in the proof of Theorem 3.1 we can assume that the traces are algebraic, and, by Theorem 2.1, we can conjugate so as to get a representation with algebraic coefficients. We now proceed as before, the important point being that the work of Weisfeiler and Nori (see [6] in this setting) requires only Zariski dense to get the conclusion of Theorem[2.9. In the case when the group is not Zariski dense, we argue as in the proof of Theorem 2.1, using the orthogonal group leaving the totally geodesic submanifold invariant. 


\section{ACKNOWLEDGMENTS}

The second author wishes to thank F. Voloch for some helpful conversations on the Tarski-Seidenberg Principle.

\section{REFERENCES}

[1] H. Bass, Groups of integral representation type, Pacific Journal of Math. 86, No. 1 (1980) 15 - 50. MR 82c:20014

[2] J. Bochnak, M. Coste, and M-F. Roy, Real Algebraic Geometry, Ergebnisse Math., vol. 36., Springer, Berlin-Heidelberg-New York, 1998. MR 2000a:14067

[3] S. S. Chen and L. Greenberg, Hyperbolic Spaces, in Contributions to Analysis, Academic Press, 1974. MR 51:13934

[4] G.J. Janusz, Algebraic Number Fields, Academic Press, 1973. MR 51:3110

[5] D. L. Johnson and J.J. Millson, Deformation spaces associated to compact hyperbolic manifolds, in Discrete Groups in Geometry and Analysis (R. Howe, ed), Progress in Math. 67 (Birkhauser 1987), 48-106. MR 88j:22010

[6] D.D. Long and A.W. Reid, Constructing hyperbolic manifolds which bound geometrically, Math. Research Letters 8 (2001), 443-456. MR 2002f:57073

[7] D.D. Long and A.W. Reid, Simple quotients of hyperbolic 3-manifold groups, Proceedings of the AMS, 126 (1998), 877-880. MR 98e:57022

[8] J. W. Morgan, Group actions on trees and the compactification of the space of $\mathrm{SO}(n, 1)$ representations, Topology, 25 (1986), 1-33. MR 87h:20062

[9] W.D. Neumann and A.W. Reid, Amalgamation and the invariant trace field of a Kleinian group, Math. Proc. Cambridge Philos. Soc. 109 (1991) 509 - 515. MR 92b:30053

[10] M.V. Nori, 'On subgroups of $\mathrm{GL}_{n}\left(F_{p}\right)$, Invent. Math. 88 (1987), 257 - 275 MR 88d:20068

[11] O.T. OMeara, Introduction to Quadratic Forms, Grundlehren der mathematischen Wissen, 117, Springer-Verlag, 1971. MR 50:269

[12] M.S. Raghunathan, 'Discrete Subgroups of Lie Groups, Ergebnisse Math., vol. 68., Springer, Berlin-Heidelberg-New York, 1972. MR 58:22394a

[13] B. Weisfeiler, Strong approximation for Zariski dense subgroups of semi-simple algebraic groups, Annals of Math. 120 (1984), 271 - 315. MR 86m:20053

Department of Mathematics and Computer Science, Emory University, Atlanta, Georgia 30322

E-mail address: emh@mathcs.emory.edu

Department of Mathematics, University of Texas, Austin, Texas 78712

E-mail address: areid@math.utexas.edu 